Electronic Supporting Information

\title{
Hafnium Doped Ceria Nanocomposite Oxide as a Novel Redox Additive for Three-way Catalysts
}

\author{
Benjaram M. Reddy, 'Pankaj Bharali, ${ }^{\text {a }}$ Pranjal Saikia, ${ }^{\text {a }}$ Ataullah Khan, \\ Stéphane Loridant, ${ }^{b}$ Martin Muhler, ${ }^{c}$ and Wolfgang Grünert ${ }^{c}$ \\ ${ }^{a}$ Inorganic and Physical Chemistry Division, Indian Institute of Chemical \\ Technology, Hyderabad - 500 007, India \\ ${ }^{b}$ Institut de Recherches sur la Catalyse-CNRS, 2 Avenue Einstein, 69626 \\ Villeurbanne Cedex, France \\ ${ }^{c}$ Lehrstuhl für Technische Chemie, Ruhr-Universität Bochum, D - 44780 \\ Bochum, Germany
}




\section{Experimental Section}

The $\mathrm{Ce}_{\mathrm{x}} \mathrm{Hf}_{1-\mathrm{x}} \mathrm{O}_{2}\left(\mathrm{CH} ; 8: 2\right.$ mole ratio based on oxides) and $\mathrm{Ce}_{\mathrm{x}} \mathrm{Zr}_{1-\mathrm{x}} \mathrm{O}_{2}(\mathrm{CZ} ; 1: 1$ mole ratio based on oxides) samples were prepared by adopting a co-precipitation method. In a typical procedure, to prepare $\mathrm{CH}$ sample, $48.8 \mathrm{~g}$ of ammonium cerium(IV) nitrate (Loba Chemie, GR grade) and $7.1 \mathrm{~g}$ of hafnium(IV) chloride (Aldrich, AR grade) were dissolved separately in $200 \mathrm{~mL}$ deionized water and mixed together. In a similar way to make $\mathrm{CZ}$ sample, $37.2 \mathrm{~g}$ of ammonium cerium(IV) nitrate (Loba Chemie, GR grade) and $29.1 \mathrm{~g}$ of zirconium(IV) nitrate (Fluka, AR grade) were dissolved separately in $200 \mathrm{~mL}$ deionized water and mixed together. Finally, the mixture solutions were diluted to $4000 \mathrm{~mL}$ with deionized water and dilute aqueous ammonia was added drop-wise with vigorous stirring until the precipitation was complete $(\mathrm{pH} \sim 8.5)$. The obtained precipitates were filtered off, washed thoroughly until free from anion impurities, dried at $393 \mathrm{~K}$ for $12 \mathrm{~h}$, and finally calcined at $773 \mathrm{~K}$ for $5 \mathrm{~h}$ in air atmosphere. The finished samples were once again thermally treated at $1073 \mathrm{~K}$ for $5 \mathrm{~h}$ in air atmosphere to investigate their temperature stability.

The X-ray powder diffraction patterns were obtained on a Siemens D-5005 diffractometer using $\mathrm{Ni}$-filtered $\mathrm{Cu} \mathrm{K} \alpha(0.15418 \mathrm{~nm})$ radiation source. The XRD phase identification was carried out with the help of Powder Diffraction File-International Center for Diffraction Data (PDF-ICDD). The average crystallite size of the oxide phases was estimated employing Scherrer equation and considering the XRD data of all prominent lines. The lattice parameter was calculated by a standard cubic indexation method using the intensity of the most prominent peak (111). The BET surface area measurements were made on a Micromeritics Gemini 2360 instrument by $\mathrm{N}_{2}$ physisorption at liquid $\mathrm{N}_{2}$ temperature. 
Prior to analysis, the samples were oven dried at $393 \mathrm{~K}$ for $12 \mathrm{~h}$ and flushed with Argon gas for $2 h$.

Raman spectra were recorded with a LabRam HR spectrometer (Jobin Yvon) equipped with a CCD detector. The emission line at $514.53 \mathrm{~nm}$ from an $\mathrm{Ar}^{+}-\mathrm{Kr}^{+}$ion $\mathrm{RM} 2018$ laser (Spectra Physics) was focused on the sample under the microscope, with the diameter of the analyzed spot being $\sim 1 \mu \mathrm{m}$. The power of the incident beam on the sample was typically 0.5 $\mathrm{mW}$. The time of acquisition was adjusted according to the intensity of the Raman scattering. The wavenumber values reported from the spectra are accurate to within $1 \mathrm{~cm}^{-1}$. In order to ascertain the homogeneity of the samples, spectra were recorded at various points and compared.

The OSC of the catalysts was determined by thermogravimetry (TG) under cyclic thermal treatments in the temperature range 573-1073 K. The weight change during the cyclic heat treatments was monitored under flowing argon or dry air. For this purpose, a commercial Netzsch TG-DTA analyzer (Luxx, STA, 409 PC, Germany) was employed. The cyclic treatments consisted of heating the sample from room temperature to $1073 \mathrm{~K}$, cooling to $423 \mathrm{~K}$, and again heating to $1073 \mathrm{~K}$ without any interruption. All heating and cooling rates were $5 \mathrm{~K} \mathrm{~min}^{-1}$. The weight loss of the sample during the second heating cycle was used to measure the oxygen release property $(\delta)$. This technique of OSC evaluation is essentially similar to that reported earlier in the literature.

$\mathrm{CO}$ oxidation was conducted in a fixed-bed micro-reactor under normal atmospheric pressure at $300-773 \mathrm{~K}$ under a heating ramp of $5 \mathrm{~K} \mathrm{~min}^{-1}$. About $100 \mathrm{mg}$ catalyst sample 
(250-355 $\mu \mathrm{m}$ sieve fraction) diluted with quartz particles of the same sieve fraction was placed in a quartz reactor for evaluation. Temperature was measured directly at the catalyst bed, using a thermocouple placed in the hollow shaft of the reactor. The following gases and gas mixtures were used (supplied by Air Liquide): argon (>99.999\% purity), $9.98 \%$ CO in argon (CO purity, >99.997\%; argon purity, >99.99\%), and 10.2\% $\mathrm{O}_{2}$ in argon (oxygen purity, >99.995\%). The total flow rates maintained by three mass flow controllers were in the range of 50-100 NmL min ${ }^{-1}$ (milliliters normalized to $273.15 \mathrm{~K}$ and 1 atmosphere). The CO and $\mathrm{CO}_{2}$ gas concentrations were measured using an Uras 14 infrared analyzer module, and the $\mathrm{O}_{2}$ concentration was measured using a Magnos 16 analyzer (Hartmann \& Braun). Prior to oxidation of $\mathrm{CO}$, the catalysts were heated to $773 \mathrm{~K}$ in $10.2 \% \mathrm{O}_{2} / \mathrm{Ar}$ gas mixture, using a heating ramp of $10 \mathrm{~K} \mathrm{~min}^{-1}$, and kept at the final temperature for $1 \mathrm{~h}$. The oxidized sample was then purged in argon and cooled to the desired starting temperature. The $\mathrm{CO} / \mathrm{O}_{2}$ reactant feed ratio was 1 , and partial pressures of $\mathrm{CO}$ and $\mathrm{O}_{2}$ were in the range of $10 \mathrm{mbar}$.

The TPR experiments were conducted in the same experimental setup used for the CO oxidation reaction. The concentration of $\mathrm{CO}$, flow rate and the heating rate were balanced with the amount of catalyst (100-150 mg) for TPR experiments as per the literature. Measurements were made using 9.98\% CO/Ar gas mixture at a flow rate of $20 \mathrm{NmL} \mathrm{min}^{-1}$ and up to $873 \mathrm{~K}$ maximum temperature with a heating rate of $5 \mathrm{~K} \mathrm{~min}^{-1}$. Before measurements, the catalysts were pretreated at $773 \mathrm{~K}$ for $1 \mathrm{~h}$ in $10.2 \% \mathrm{O}_{2} / \mathrm{Ar}$ gas mixture, using a heating rate of $10 \mathrm{~K} \mathrm{~min}^{-1}$. The oxidized sample was then purged in argon and cooled to the desired starting temperature. 